\title{
A simplified laparoscopic-assisted gastrostomy technique: a single center experience
}

\author{
Hesham M. Sheir , Tamer A. Wafa, Abdelrahman Elshafey and Mohamed Elzohiri
}

\begin{abstract}
Background: Although percutaneous endoscopic gastrostomy (PEG) is considered simple and effective, major complications have been reported because of the partly blind placement technique. Laparoscopic gastrostomy was described to overcome most of the problems and the contraindications of PEG. Various modifications have been reported to anchor the stomach to the abdominal wall.

This study aims at highlighting a single center experience using a simplified technique for gastrostomy and evaluates its outcome.

Results: The age of the patients ranged from 7 days to 3 years (mean $11.75 \pm 12$ months in group A and $16 \pm 3$ months in group B). The mean body weight at time of gastrostomy was $6.5 \pm 4.6 \mathrm{~kg}$ in group A and $7.5 \pm 2$ in group B. The mean operative time was $24.8 \pm 4$ min in group A and $25 \pm 1.6$ in group B, ranging from 18 to 31 min. The incidence of gastrostomy-related complications was 20\% (three cases) in group A versus 12.5\% (one case) among patients included in group B. Yet, this difference was statistically insignificant ( $p=0.651$ ). No major complications were reported apart from persistent gastrocutaneous fistula in one out of eight cases followed after removal of the gastrostomy (12.5\%). There is no significant difference in the outcome either in neonates or in patients less than $5 \mathrm{~kg}$.
\end{abstract}

Conclusions: The described simple technique of laparoscopic-assisted gastrostomy is easy, effective, and with a very low incidence of complications. It is also equally safe in neonates and children less than $5 \mathrm{~kg}$.

Keywords: Laparoscopic-assisted, Gastrostomy, PEG

\section{Background}

Gastrostomy is a widely performed procedure for several indications in pediatrics [1]. Percutaneous endoscopic gastrostomy (PEG) is simple and effective, and so it is considered the method of choice in many centers [2]. However, many minor and even major complications have been reported after PEG because of the partly blind placement technique [3]. Laparoscopic gastrostomy was described to overcome most of the problems and the contraindications of PEG with the advantages of allowing proper visualization and selection of the gastrostomy site and avoiding visceral injury [4, 5]. Various modifications have been reported to anchor the stomach to the abdominal wall in order to improve the

\footnotetext{
* Correspondence: heshamsheir@yahoo.com

Mansoura University Children's Hospital, Mansoura University, 60 Gomhoria St, Mansoura 35516, Egypt
}

effectiveness and minimize the complications of the procedure $[6,7]$. A simplified laparoscopic-assisted gastrostomy procedure was adopted at Pediatric Surgery Department, Mansoura University, since May 2016 in order to perform the gastrostomy procedure simply and safely.

\section{Aim of the work}

This study aims at highlighting a single center experience using a simplified laparoscopic-assisted gastrostomy technique with evaluation of its feasibility, outcome, and complications.

\section{Methods}

This is a retrospective study conducted at Pediatric Surgery Department, Mansoura University. The study was performed by reviewing patients' medical records during the period from May 2016 to December 2017. All 
patients that underwent gastrostomy with the simplified laparoscopic-assisted technique were included in the study, whereas all gastrostomies performed by other techniques were excluded.

The study protocol was accepted by Institutional Research Board (R.18.03.108). After informed consent is signed by the legal guardian, the simplified laparoscopicassisted gastrostomy technique was performed as described by Rothenberg (1999) [7] with slight modifications: A $5 \mathrm{~mm}$ camera port is inserted at the umbilicus, in addition to a $5 \mathrm{~mm}$ port introduced at the gastrostomy site in the left upper quadrant of the abdomen, between the umbilicus and the costal margin, with flexible modification according to the body configuration of the patient. A grasper is introduced through the trocar to grasp the gastrostomy site which is selected properly under vision (Fig. 1a). The grasped gastric wall is pulled through the trocar which is removed to allow the gastrostomy site to be exteriorized outside the abdomen via the abdominal incision performed for port introduction (Fig. 1b). Two stay sutures anchor the anterior gastric wall, and a purse-string suture is inserted in the exposed stomach (Fig. 1c). Then, four to eight sutures are taken between the stomach and the anterior rectus sheath or the external oblique aponeurosis to secure fixation of the gastrostomy site to the anterior abdominal wall (Fig. 1d). Finally, the stomach is incised at the middle of the purse-string suture to allow introduction of the gastrostomy tube (Fig. 1e). All sutures are tied, the balloon of the gastrostomy tube is inflated, and the final view is checked laparoscopically (Fig. If and g).
Twenty-three patients were included in the study and were divided into two groups:

- Group A included 15 cases performed gastrostomy alone.

- Group B included eight cases where gastrostomy is added to fundoplication.

The medical records of all selected cases were reviewed for the age and the body weight at time of operation, the indication for gastrostomy, the operative time, and the complications of the procedure.

Data were analyzed with statistical package for social science (SPSS) version 22 (SPSS Inc., Chicago, Illinois, USA). The normality of data was first tested with Shapiro test. Qualitative data were described using number and percent. Continuous variables were presented as mean \pm SD (standard deviation). Student $t$-test was used to compare two groups with the threshold of significance is fixed at $5 \%$ level ( $p$ value).

\section{Results}

The present study included 23 patients underwent laparoscopic-assisted gastrostomy: 15 of them were males $(65.2 \%)$ and 8 were females (34.8\%). Gastrostomy was performed because of neurological problems in ten cases $(43.5 \%)$, being the commonest indication for gastrostomy in the current study followed by esophageal atresia representing the indication in $30.4 \%$ of cases (Table 1).

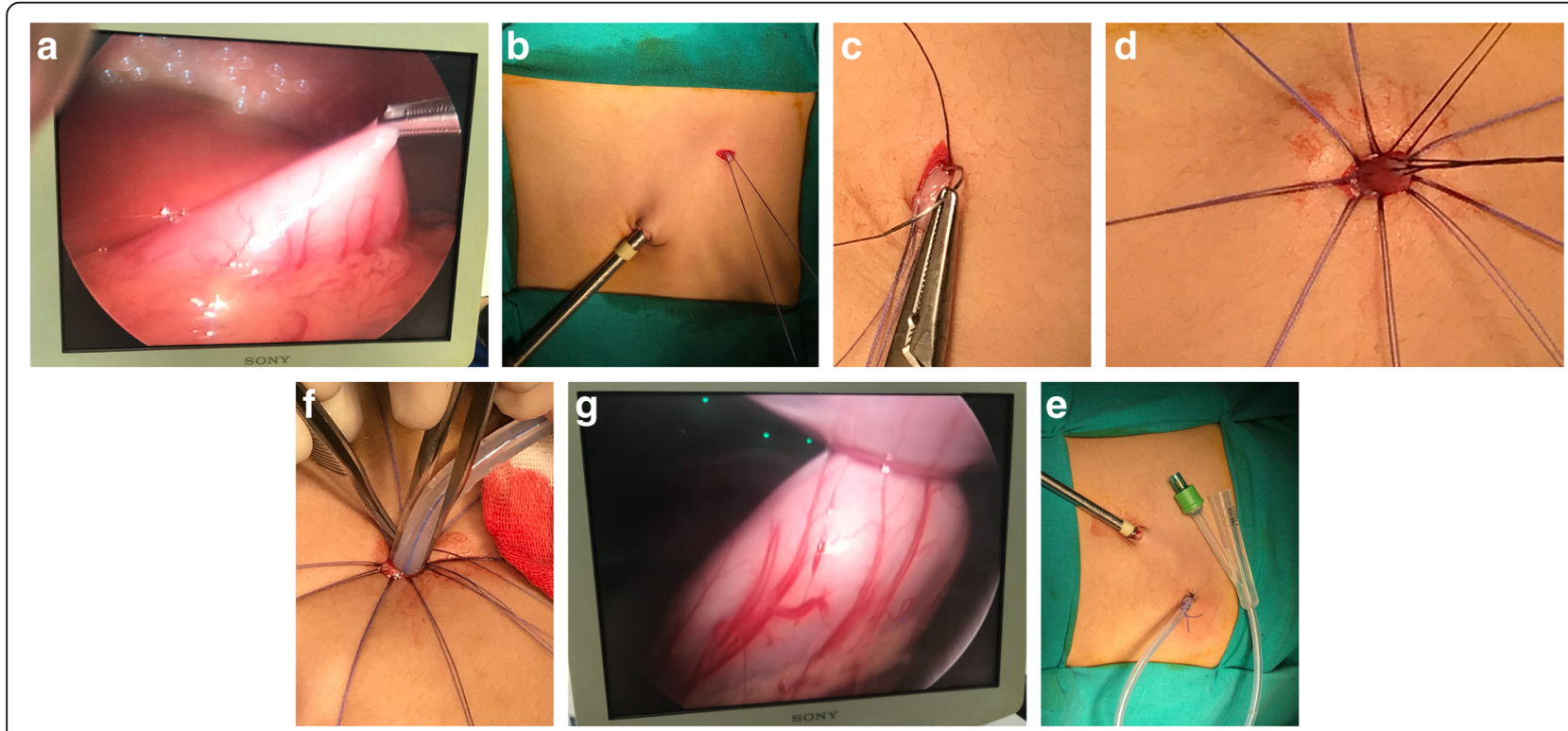

Fig. 1 Steps of laparoscopic-assisted gastrostomy: a Grasping the selected gastrostomy site laparoscopically. b The grasped stomach exteriorized after trocar removal and anchored by stay sutures. c A purse-string suture is inserted in the exposed stomach. $\mathbf{d}$ Exteriorized gastric wall is fixed to the anterior abdominal wall. e Gastrostomy tube insertion. $\mathbf{f}$ Laparoscopic check after gastrostomy insertion. $\mathbf{g}$ Final view 
Table 1 Indications for gastrostomy

\begin{tabular}{lll}
\hline Indication & Number of cases & Percentage \\
\hline Neurological problems & 10 & $43.5 \%$ \\
Esophageal atresia & 7 & $30.4 \%$ \\
Post-corrosive injury & 4 & $17.4 \%$ \\
$\begin{array}{l}\text { Instrumental trauma during } \\
\text { foreign body extraction }\end{array}$ & 2 & $8.7 \%$ \\
\hline
\end{tabular}

The gastrostomy was performed at ages ranged from 7 days to 3 years, with the mean age $11.75 \pm$ 12 months in group $\mathrm{A}$ and $16 \pm 3$ months in group $B$, with no statistically significant difference between both groups $(p=0.34)$. The procedure was performed in the neonatal period in seven cases $(30.4 \%$ of all cases). The body weight at the time of the operation ranged from 1.8 to $14 \mathrm{~kg}$ with the mean body weight $6.5 \pm 4.6 \mathrm{~kg}$ in group $\mathrm{A}$ and $7.5 \pm 2$ in group B. On comparing both groups, there was no statistically significant difference regarding body weight $(p=0.585)$, and nine cases were less than $5 \mathrm{~kg}$ at time of performing the gastrostomy (39.1\% of all cases).

Gastrostomy was performed alone in 15 cases (65.2\%) (group A) and with fundoplication in 8 cases $(34.8 \%)$ (group B). The operative time needed to complete the procedure ranged from 18 to $31 \mathrm{~min}$, and the mean was $24.8 \pm 4 \mathrm{~min}$ in group $\mathrm{A}$ and $25 \pm$ 1.6 in group B where the time needed for fundoplication was omitted. There was no significant difference between both groups ( $p=0.893)$.

Follow-up period ranged from 3 to 12 months with the mean follow-up period $6.3 \pm 3$ months in group $\mathrm{A}$ and $5.3 \pm 2$ in group $\mathrm{B}(p=0.424)$. The incidence of gastrostomy-related complications was $20 \%$ (three cases) in group A versus $12.5 \%$ (one case) among patients included in group B. Yet, this difference was statistically insignificant $(p=0.651)$. There were no reported complications related to fundoplication in group $B$.

Most of the reported complications were minor complications that were managed without further surgical interventions. In group $\mathrm{B}$, the single reported complication was infection around the gastrostomy site with granuloma formation, which was managed by local antibiotics with a barrier cream. Similarly in group A, one case was complicated by granuloma; however, this case was later on complained from leakage of the gastric contents around the gastrostomy tube, and this was managed by switching to a smaller tube to give the chance for the stoma to shrink, and then a tube of the same previously introduced diameter was reintroduced. Tube dislodgment was reported in one case in group $\mathrm{A}$, but fortunately another tube was reintroduced rapidly without further complications. Moreover, persistent gastrocutaneous fistula that will need surgical closure was reported in another case. By considering that eight cases only underwent gastrostomy tube removal and properly followed up for more than 2 months, so the incidence of persistent gastrocutaneous fistula after removal of the gastrostomy tube was $12.5 \%$ in this small case series. However, a longer follow-up period and a larger number of cases are needed to achieve more accurate incidence of such complication.

There was no significant difference in the outcome of the simplified laparoscopic-assisted gastrostomy in the cases that were operated at age of 1 month or less and those that were operated at older age. The mean operative time was $26.43 \pm 3.36 \mathrm{~min}$ in the neonatal group versus $24.19 \pm 3.1 \mathrm{~min}$ in the older group $(p=0.09)$, and only one minor complication was reported in the neonatal group (14.3\%) versus three cases with complications in the older group (18.8\%). Moreover, by considering the body weight at the time of the procedure, the mean operative time was $26.22 \pm 3 \mathrm{~min}$ in patients who were $5 \mathrm{~kg}$ or less and was $24 \pm 0.86 \mathrm{~min}$ in patients who were over $5 \mathrm{~kg}(p=0.54)$. The incidence of complications was $11.1 \%$ in the group with lower body weight, while it was $21.4 \%$ in the other group (Fig. 2).

\section{Discussion}

Among several indications of gastrostomy in children, chronic neurological problems causing feeding difficulties represent the most common indication with incidences ranging from 40 to $75 \%$ [8-10]. Similarly, neurological abnormalities (mainly cerebral palsy) were the indication of gastrostomy in $43.5 \%$ of the cases included in the present study. The mean age at the time of the procedure was $11.75 \pm 12$ months in group $\mathrm{A}$ and 16 \pm 3 months in group $B$, which are younger than the mean ages reported by most of the other studies ranging from 2 to 4.5 years $[10,11]$. This is may be due to the large number of gastrostomies performed for cases of esophageal atresia at neonatal period in this study.

Using a minimally invasive approach became the standard practice in performing gastrostomy in children. PEG gained wide popularity with the advantages of being performed with minimal anesthesia and followed by rapid recovery and short hospital stays [2]. Yet, major complications and even procedure-related mortality were reported after PEG $[8,12]$. So, the laparoscopic approach is aiming to combine the simplicity of PEG with the safety of the open approach. Laparoscopy allows ideal visualization and proper selection of the gastrostomy site, but the details about the method of introduction of the gastrostomy device vary widely, following either an intracorporeal Seldinger technique or an extracorporeal insertion approach [13]. In the present study, a simplified laparoscopic-assisted extracorporeal approach is evaluated for its safety and effectiveness. 


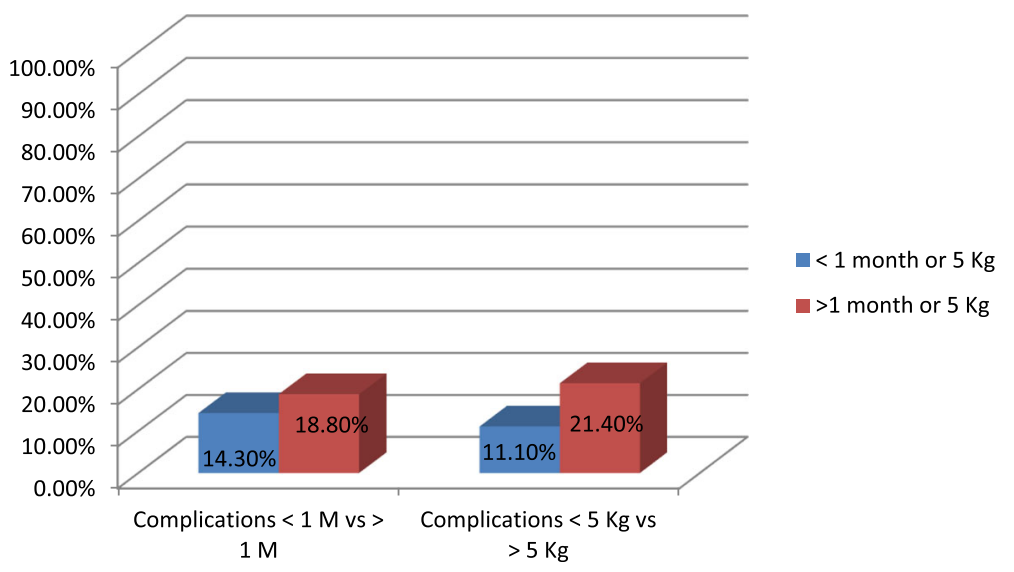

Fig. 2 Comparison of complications in patients $<1$ month versus $>1$ month and $<5 \mathrm{~kg}$ versus $>5 \mathrm{~kg}$ (SEM for age 0.99 and for body weight 4.14)

The mean operative time in this study was $24.87 \pm$ 3.28 min. This is comparable with the results of Rothenberg et al. (1999) [7] (15 min) who used a similar technique and Tomicic et al. (2002) [11] (33.6 \pm 14.3$)$, who used the Seldinger maneuver. Mizrahi et al. (2014) reported a much longer operative time with a mean $76.8 \pm$ 7 min [14]. This is because they performed the two purse-string sutures completely intracorporeal.

Previous meta-analysis concluded that laparoscopic approach is safer than PEG because of its clear visualization that prevent bowel injury, and it also allows immediate detection and correction of any major complications [15]. In the present study, complications were reported in four cases (17.4\%): three of them were minor complications $(13 \%)$ whereas major complications were reported only in one case (4.3\%). This overall complication rate is within the reported rates ranging from 3 to $60 \%[2,16,17]$. This wide range is due to the wide variability in the gastrostomy techniques and the different considerations of the minor complications. Kim et al. (2017) compared the outcome after endoscopic, laparoscopic, and open gastrostomy, and they found no significant difference between different techniques. They reported minor complications in $46.8 \%$ of cases performed laparoscopic gastrostomy with Seldinger technique and major complications in $8.9 \%$ [2]. This complication rate is much higher than that reported in the present study. We believe that the simplified extracorporeal approach used in this study had a role in preventing major complications. This is supported by the $0 \%$ incidence of major complications reported by others performing similar extracorporeal technique [7, 17].

Persistent gastrocutaneous fistula is considered a major complication by some authors and was reported with an incidence up to $60 \%$ after surgically inserted gastrostomy whether open or laparoscopic, and this was statistically significant when compared with the endoscopic insertion group [2]. The incidence of this complication was $12.5 \%$ in the present study, but many gastrostomies were not removed yet, so longer follow-up is required.

In the present study, the complication rate was even lower in patients performed gastrostomy with fundoplication (group B) with no significant difference when compared with cases performed gastrostomy alone (group A) $(p=0.6)$. Although Acroyd et al. (2011) reported higher incidence of complications in the fundoplication group (19.4\%) in comparison with children who underwent gastrostomy alone (15.3\%), yet this difference was not statistically significant $(p=0.1)$ [15]. Moreover, in the current study, laparoscopic-assisted gastrostomy was found safe with comparable outcome among neonates and children less than $5 \mathrm{~kg}$.

Finally, we admit the limitations of this study regarding the retrospective study design, the small number of cases, and the short follow-up period. So, further prospective studies are recommended bases on our initial results.

\section{Conclusion}

The simplified laparoscopic-assisted gastrostomy technique is easy to perform, with a very low incidence of complications. The extracorporeal laparoscopic-assisted approach increased the safety and the simplicity of the procedure and ensure proper fixation of the stomach to the abdominal wall. Moreover, this technique seemed to be equally safe in neonates and infants less than $5 \mathrm{~kg}$.

Abbreviations

PEG: Percutaneous endoscopic gastrostomy; SPSS: Statistical package for social science

Acknowledgements

Not applicable. 


\section{Authors' contributions}

HS shared in designing the study protocol, was a major contributor in writing the manuscript, and shared in collection and analysis of data. TW shared in designing the study protocol, collection and analysis of data, and revision of the manuscript. AE shared in collection and analysis of data and revision of the manuscript. ME shared in collection of data and in writing the manuscript. All authors read and approved the final manuscript.

\section{Funding}

No funding sources to be declared.

\section{Availability of data and materials}

The datasets used and/or analyzed during the current study are available from the corresponding author on reasonable request.

\section{Ethics approval and consent to participate}

The study protocol and the consent form were accepted by Institutional Research Board (Faculty of Medicine, Mansoura University) (R.18.03.108). The informed written consent to participate in the study was provided by all participants (or their parent or legal guardian in the case of children under 16).

\section{Consent for publication}

Not applicable (No personal data or photos that could interfere with the patient's confidentiality).

\section{Competing interests}

The authors declare that they have no competing interests.

Received: 10 October 2019 Accepted: 12 November 2019

Published online: 03 January 2020

\section{References}

1. Rahnemai-Azar AA, Rahnemaiazar AA, Naghshizadian R, Kurtz A, Farkas DT. Percutaneous endoscopic gastrostomy: indications, technique, complications and management. World J Gastroenterol. 2014;20(June): 7739-51.

2. Kim J, Koh H, Chang EY, Park SY, Kim S. Single center experience with gastrostomy insertion in pediatric patients: a 10-year review. Pediatr Gastroenterol Hepatol Nutr. 2017;20(1):34-40.

3. Fascetti-Leon F, Gamba P, Dall'Oglio L, Pane A, dé Angelis GL, Bizzarri B, Fava G, Maestri L, Cheli M, Di Nardo G, La Riccia A, Marrello S, Gandullia P, Romano C, D'Antiga L, Betalli P. Complications of percutaneous endoscopic gastrostomy in children: results of an Italian multicenter observational study. Dig Liver Dis. 2012;44:655-9.

4. Bankhead RR, Fisher CA, Rolandelli RH. Gastrostomy tube placement outcomes: comparison of surgical, endoscopic, and laparoscopic methods. Nutr Clin Pract. 2005:20:607-12.

5. Matuszczak E, Komarowska M, Dębek W, Kondej-Muszyńska K, Siergiejko G, Hermanowicz A. Laparoscopic assistance enables percutaneous endoscopic gastrostomy (PEG) in patients after prior abdominal and gastric surgery. Case series and review of the literature. Pediatria Polska. 2016;91:547-51.

6. Georgeson KE. Laparoscopic gastrostomy and fundoplication. Pediatr Ann. 1993:22:675-7.

7. Rothenberg SS, Bealer JF, Chang JHT. Primary laparoscopic placement of gastrostomy buttons for feeding tubes: a safer and simpler technique. Surg Endosc. 1999;13:995-7.

8. Sheehan JJ, Hill AD, Fanning NP, Healy C, McDermott EW, O'Donoghue DP, O'Higgins NJ. Percutaneous endoscopic gastrostomy: 5 years of clinical experience on 238 patients. Irish Med J. 2003;96(9):265-7.

9. Peter SD, Holcomb GW. Gastroesophageal reflux disease and fundoplication in infants and children. Annals Pediatr Surg. 2007;3(1):1-10.

10. Viktorsdóttir MB, Óskarsson K, Gunnarsdóttir A, Sigurdsson L. Percutaneous endoscopic gastrostomy in children: a population-based study from Iceland, 1999-2010. J Laparoendosc Adv Surg Tech A. 2015;25(3):248-51.

11. Tomicic JT, Luks Fl, Shalon L, Tracy TF. Laparoscopic gastrostomy in infants and children. Eur J Pediatr Surg. 2002;12(2):107-10.

12. Vervloessem D, van Leersum F, Boer D, Hop WC, Escher JC, Madern GC, de Ridder L, Bax KN. Percutaneous endoscopic gastrostomy (PEG) in children is not a minor procedure: risk factors for major complications. Semin Pediatr Surg. 2009;18(2):93-7.
13. Baker L, Emil S, Baird R. A comparison of techniques for laparoscopic gastrostomy placement in children. J Surg Res. 2013;18(2):93-7.

14. Mizrahi I, Garg M, Divino CM, Nguyen S. Comparison of laparoscopic versus open approach to gastrostomy tubes. JSLS. 2014;18:28-33.

15. Acroyd R, Saincher M, Cheng S, El-Matary W. Gastrostomy tube insertion in children: the Edmonton experience. Can J Gastroenterol. 2011;25(5):265-8.

16. Suksamanapun N, Mauritz FA, Franken J, van der Zee DC, van HerwaardenLindeboom M. Laparoscopic versus percutaneous endoscopic gastrostomy placement in children: results of a systematic review and meta-analysis. J Minim Access Surg. 2017;13(2):81-8.

17. Merli L, De Marco EA, Fedele C, Mason EJ, Taddei A, Paradiso FV, Catania VD, Nanni L. Gastrostomy placement in children: percutaneous endoscopic gastrostomy or laparoscopic gastrostomy? Surg Laporosc Endosc Percutan Tech. 2016;26(5):381-4.

\section{Publisher's Note}

Springer Nature remains neutral with regard to jurisdictional claims in published maps and institutional affiliations.

\section{Submit your manuscript to a SpringerOpen ${ }^{\circ}$ journal and benefit from:}

- Convenient online submission

- Rigorous peer review

- Open access: articles freely available online

High visibility within the field

- Retaining the copyright to your article

Submit your next manuscript at $\boldsymbol{\nabla}$ springeropen.com 